\title{
Self-organized and Context-Adaptive Information Diffusion in Vehicular Ad Hoc Networks
}

\author{
Christian Adler*, Stephan Eichler ${ }^{\dagger}$, Timo Kosch ${ }^{\ddagger}$, Christoph Schroth ${ }^{\dagger}$ and Markus Strassberger ${ }^{\ddagger}$ \\ *Institute for Informatics, University of Munich, Munich, Germany \\ ${ }^{\dagger}$ Institute of Communication Networks, Technische Universität München, Munich, Germany \\ ${ }^{\ddagger}$ BMW Group Research and Technology, Munich, Germany
}

\begin{abstract}
Vehicular ad hoc networks are considered as technology enabler for new automotive telematics services. In particular, those networks will enable foresighted active safety applications. However, in order to guarantee fast market introduction, a large variety of different applications with different traffic patterns must be supported. Due to the strict limitation of the available bandwidth in ad hoc networks, it is likely that in many situations the channel capacity is not sufficient to satisfy all transmission requests of all vehicles. However, a situation adaptive and self-organized utilization of the ad hoc network can optimize the overall utility of the deployed applications in the participating vehicles. Thereby, channel access is coordinated in a way that those data packets can access the channel first that provide the biggest expected utility for other vehicles in a specific situation. In this paper we delineate the effects of this implicit and situation adaptive information diffusion strategy and show that neither an explicit dissemination area or message life time is necessary.
\end{abstract}

\section{INTRODUCTION}

In the last couple of years, a lot of effort was put into the development of vehicular ad hoc networks (VANETs) as technology enabler for new telematics services, ranging from road safety to traffic information and mobile payment. The cooperation of vehicles is widely considered as one key issue in the visionary field of accident-free driving. Cooperative and foresighted driving require a large amount of relevant information to be distributed among the vehicles. In the context of those safety-related applications in VANETs, data is therefore mostly spread among vehicles in a broadcast fashion. However, the available wireless bandwidth is strictly limited and must therefore be used carefully. In addition, the information diffusion strategy must also ensure that no important information is lost in case that there are currently only very few equipped vehicles in a certain area.

In order to use the available bandwidth as optimal as possible in any situation, we follow the principle of network utility maximization (NUM) [1], [2] and self-organization. In the domain of VANETs, taking into account the utility of a data packet that should be transmitted has already been addressed by Wischhof et al. [3], [4] and is (from a more generic point of view) formalized by Kosch [5].

In the following, we further quantify the concept of utility maximization by taking into account individual drivers' interest in certain information. In addition, we show that a utility-based information diffusion strategy also meets the requirements of self-organization and situation adaptivity with regard to implicit dissemination areas, message life-times, and re-broadcasting intervals. Self-organization can effectively be realized by assigning the responsibility of message forwarding to the forwarding nodes instead of the originator. A continuous re-evaluation at receiver side allows for a flexible and situation-aware diffusion strategy. Note that originator centric approaches like the ones proposed in [6]-[8] impose high vulnerability for denial-of-service attacks. Inadequately large dissemination areas or long message life-times can easily lead to holding a large number of unnecessary packets in the network, which in turn will lead to a network collapse. In order to prevent such attacks, a re-evaluation at the receiver side concerning the plausibility of a further packet relay has to be accomplished. A receiver driven packet relay implicitly addresses this issue.

In this paper we introduce new concepts for efficient and beneficial message diffusion for VANETs using selforganization and message benefit determination techniques. We'll present simulation results to prove the viability and show the capabilities of these concepts.

\section{UTILITY-CENTRIC INFORMATION DIFFUSION}

According to [9], in case of a high network load limiting the number of data transmissions, nodes should evaluate the utility of all messages bound to be sent. Using these utility values to schedule message transmissions will ensure that the most relevant messsages will be relayed first, hence, optimizing the overall utility of the information diffusion. In this context it has to be explicitly distinguished between the terms utility and relevance. Utility refers to the effective benefit a vehicle has when receiving a message, whereas relevance refers to the expected overall utility for all receiving nodes.

Using utility evaluation for packet scheduling can optimize channel utilization. The channel is considered being used optimally, if a packet scheduling can be found that maximizes the overall utility of the vehicles participating in the network. Such packet sequence thereby comprises two separate steps. First, each vehicle has to order its own local message queue according to the corresponding expected utility. Second, the medium access strategy has to ensure that those vehicles can access the medium first, that currently carry the packets with the highest expected relevance.

To apply utility estimation, parameters have to be identified and categorized to determine the message benefit (Eqn. 1). 
In our work we used message age, last broadcast time, and last reception time to characterize the message context. In addition, driving direction (as proposed in [10]), vehicle speed, and roadtype are parameters of the vehicle context. The third category, information context, is including e.g. distance to information source, time-of-day, information quality, information type, and rate of change as parameters.

Eqn. 1 is a generalized form of the benefit function used to quantify the benefit of a piece of information. Supposed there are $N$ parameters such as distance to information source and message age. They all have to be computed with the help of the message content $m$, the vehicle context $v$, and the already stored messages $s$. All these $N$ parameters are evaluated with application-dependent sub-functions, which result in the values $b_{i}$. The results of the functions $b_{i}$ finally have to be weighted with application-dependent factors $a_{i}$, where the sum of all $a_{i}$ must be equal to 1 . In the context of a collision warning, for example, the message age parameter is more important for the overall message benefit than the parameter evaluating the information quality. Hence, the respective weighting factor $a_{i}$ is larger than the others. The weighting factors can be specified based on e.g. road traffic statistics or other empirical observations. In a final step of the calculation all weighted parameters have to be summed up and divided by $N$, resulting in the respective message benefit value.

$$
\begin{gathered}
\text { MessageBenefit }=\frac{1}{N} \cdot \sum_{i=1}^{N} a_{i} \cdot b_{i}(m, v, s) \\
\text { III. MEDIUM ACCESS STRATEGIES FOR } \\
\text { RELEVANCE-ORIENTED MESS AGE DISSEMINATION }
\end{gathered}
$$

As an integral part of our approach, the inter-vehicle contention for the shared medium must be adapted such that the most relevant packet all nodes within mutual communication range have available is granted access to the medium.

\section{A. Per-packet and per-period timer adaptation}

By selecting short contention windows (CW) in case a highly relevant packet requests access to the medium, the node's likelihood to win the contention process is increased. The smaller the CW, the shorter the timers defining the period of time after a node may try or retry to access the shared medium.

The effects of changing the contention window size for the purposes of traffic differentiation can be seen in Fig. 1. In this first example, three different wireless stations permanently contend for access to the shared, wireless medium. Blue rectangles visualize timers counting down, where rectangles with a checkered muster symbolize the remaining backoff- or defer times, which cannot be counted down since the medium is currently sensed to be busy. These remaining periods of time have to be transferred into the next "contention period", i.e. the next period of time between two DIFS slots of an idle medium. This transfer of remaining timers is represented by the arrows in Fig. 1. One can also clearly see the impact of reducing the contention window size for one of the stations in a neighborhood. In this example, station three is assumed to have extremely relevant data packets available. To increase the global network utility, that station should also win the contention for the shared medium with a higher likelihood than the neighboring stations. On the very left side of the figure, station three tries to access the medium, senses the medium to be idle for DIFS and starts its defer timer. Due to a reduced $\mathrm{CW}$ size, this timer is rather short: Station three quickly gets its first transmission opportunity. This qualitative analysis highlights the possibility to grant stations with highly relevant data a quicker and therefore a prioritized access to the wireless medium.

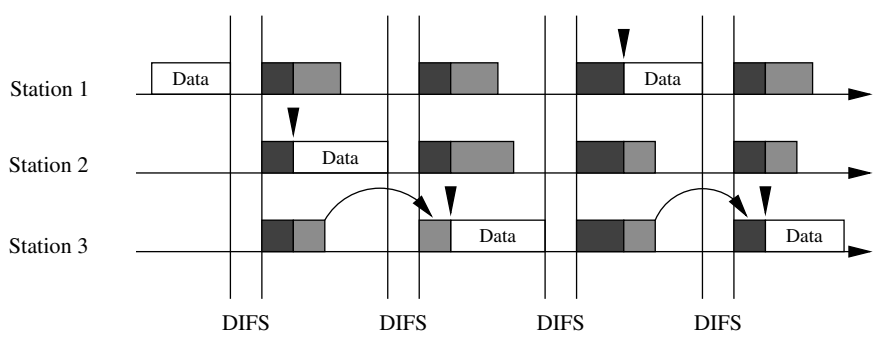

Fig. 1. Contention between the three stations with "per-packet" timer adaptation: Station three gets preferred access to the shared medium

Besides the mere adaptation of the nodes' actual CW sizes according to estimated packet relevance values, one could think about taking one step further and passing on the transfer of remaining timer values into the next contention periods. Instead, timers are newly computed for each contention period, reflecting the estimated relevance of the currently treated data packets. This helps avoiding the following situation: A node trying to transmit a rather redundant packet has initiated a relatively long timer some contention periods ago. After waiting for a sufficient amount of periods, its remaining timer is short and even may be shorter than the one started by another node which tries to transmit a highly beneficial packet. Station one in Fig. 1, for example, starts a rather long timer after sending the first packet. In the third contention period, however, it is granted access to the medium, since the remaining timer is already quite short. Despite having started a short timer, station three has to defer its medium access into the next period. Fig. 2 shows the same three wireless stations once again: Now, remaining timers are not transferred into the next contention periods, but newly computed each time the medium was idle for at least DIFS. This ensures a medium access strategy that is fully benefit-oriented and lacks any benefit-agnostic, fair resource allocation tendency. Not the time a packet has already been waiting, but its upto-date benefit value decide upon its chance to get medium access.

\section{Simulation Results}

In the following we show simulation results that proof the medium access strategy and the self-organizing character of the diffusion approach regarding dissemination areas, message lifetime and situation adaptability. 


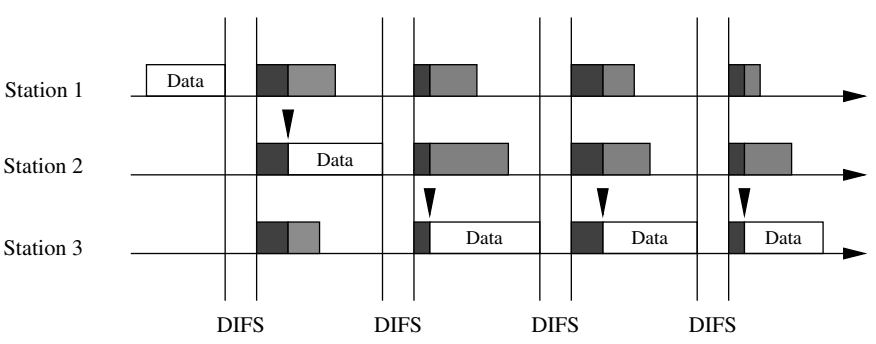

Fig. 2. Contention between the three stations with "per-period" timer adaptation

\section{A. Medium Access}

We proposed two ways of modifying the medium access functionality: First, one may consider starting defer-and backoff timers only once and transfer remaining timers (e.g., in case another node transmits on the channel) into the next so called contention periods. In this case, timers are counting down until they expire and trigger their respective nodes' transmissions. As a second possibility, the timers are newly set in each contention period according to the current $\mathrm{CW}$ size, thereby ensuring a total benefit-orientation of the medium access functionality: Not the time a packet has already been waiting for access to the shared medium, but solely its current relevance for the adjacent nodes defines its likelihood to get medium access.

Fig. 3 shows the results of a related simulation, where a typical VANET scenario has been applied: 300 wireless enabled vehicles are driving around in a $8 \mathrm{~km}^{2}$ urban area. The network load was set to 10 new packets being generated per second. Each node is assumed to have a bandwidth of $0.3 \frac{\mathrm{Mbit}}{\mathrm{s}}$ available to simulate a highly loaded network. To differentiate data traffic with regard to its current relevance, the sizes of the CWs are adapted (in the range between CWmin (31 slots) and CWmax (1023 slots)). As a consequence, defer-and backoff timers are on average longer than in case no differentiation is applied (CWmin is mostly applied then). If timers are longer, the rate of data packets getting access to the medium is smaller, since the average time spent for backing off is increased. Simulations show that the global aggregate utility achieved with the help of each of the two different medium access strategies is significantly higher than without any MAC-level traffic differentiation. Moreover, the graphs representing the global utility are rather similar for different parameter settings. However, due to space restrictions, we do not further elaborate on these results.

To examine the channel utilization in the cases of no traffic differentiation, per-packet timer adaptation and per-period timer adaptation, the global number of successfully received packets in the whole network has been tracked. As one can see in Fig. 3, the modification of the MAC functionality as introduced above always leads to a degradation of net data throughput due to the on average timer prolongation. The perperiod timer adaptation shows the minimum throughput of the two modified MAC schemes, since timers are newly started

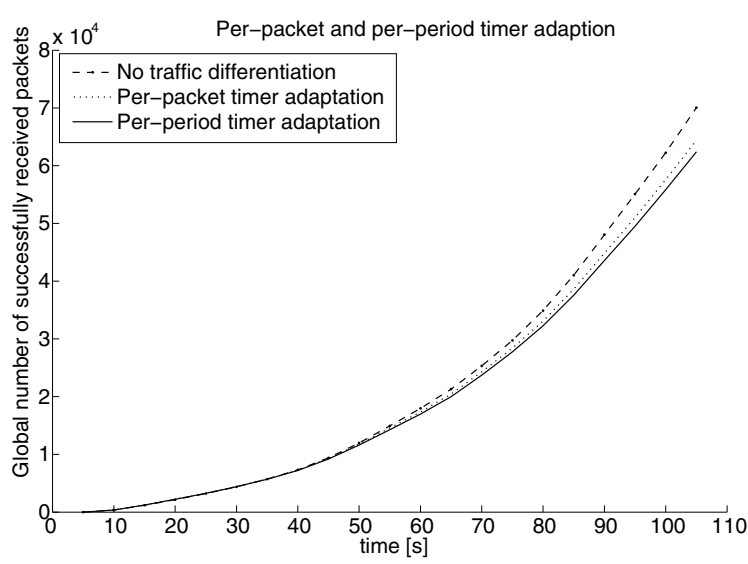

Fig. 3. Comparison of the number of successfully received packets when applying per-period and per-packet timer adaptation

in each contention period. In this way, the inter-vehicular packet schedule can be improved in comparison to the perpacket timer adaptation, but at the significant expense of data throughput. Note that although the net data throughput is lower due to the increased timer sizes in both cases (perpacket and per-period timer adaptation), the global network utility is significantly higher than without traffic differentiation. Both schemes are able to compensate for the decreased data throughput and considerably improve the global utility provided to the network.

\section{B. Dissemination Area}

An explicit determination of the dissemination area of a message is hard to realize, regarding a permanently changing situation in VANETs. Therefore, a self-organizing and contextadaptive form of dissemination areas is necessary, so that it adjusts itself to the current situation.

Fig. 4 shows a plot of all message transmissions, during $300 \mathrm{~s}$ of simulation time, on a map containing two hazards with the same impact. As it can be clearly seen, the dissemination areas of the two messages are aligned around the corresponding hazard. This is due to the weight of the distance parameter. Under the assumption that messages are more relevant the closer a vehicle is to the described hazard, Fig. 4 illustrates a reasonable formation of dissemination areas. However, a parameter incorporating the last transmission of a message ensures that both messages are sporadically distributed in both dissemination areas.

The graph in Fig. 5 represents a different view of the prioritization character of the selforganizing diffusion. Due to its higher impact, the dissemination area of message 2 swells, and therefore, reduces the dissemination area of message 1. This points out the context-adaptive character of the dissemination approach.

Fig. 6 illustrates descriptively how the formation of dissemination areas adapts to the situation. In a scenario with five hazards, the corresponding messages are distributed within 


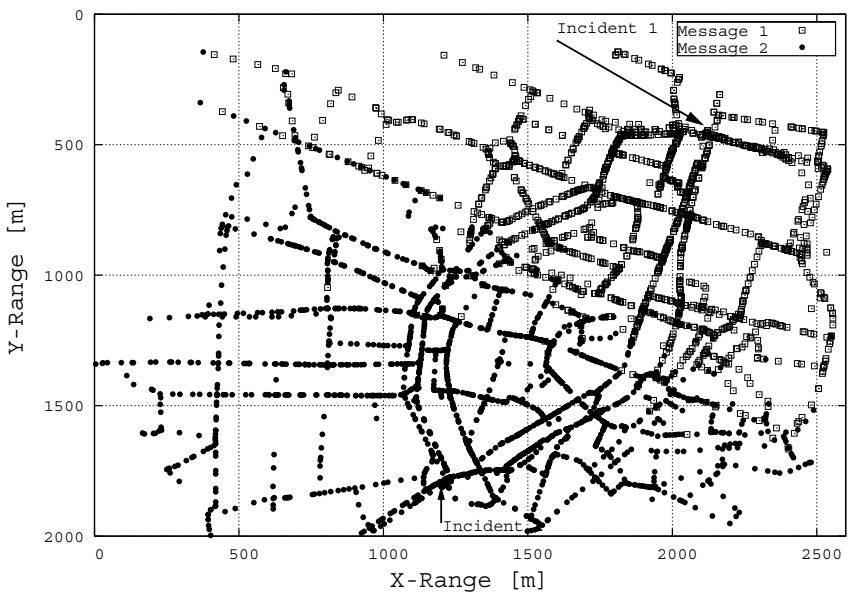

Fig. 4. Dissemination areas in a scenario with two hazards, each with an equal impact

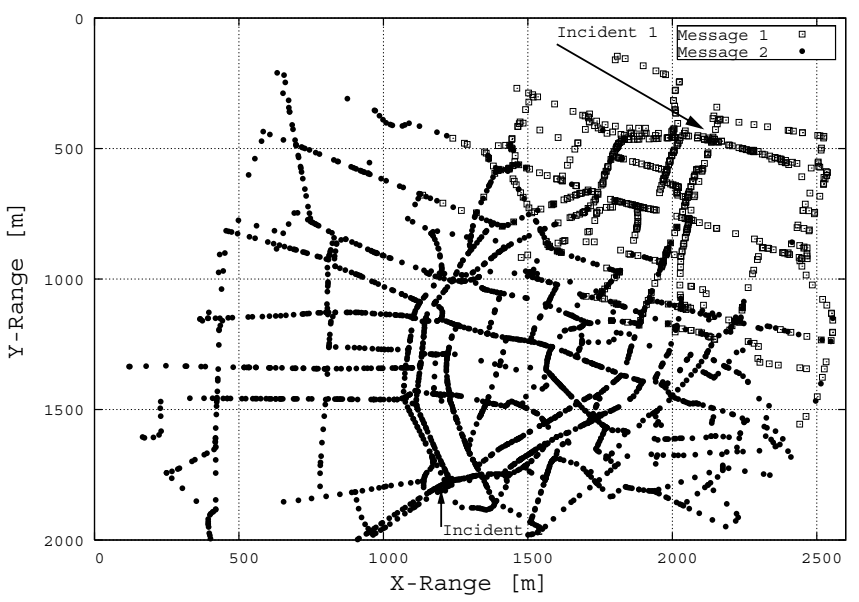

Fig. 5. Dissemination areas in a scenario with two hazards, each with a different impact

their properly delimited dissemination areas.

\section{Message Lifetime}

It is very important that a relevant message is not only flooded through the VANET once, but that it circulates within the areas of importance as long as necessary. Long message lifetimes assure that vehicles entering these areas are also informed later in time. As a result, it is necessary to rebroadcast a message from time to time. Because there will always be a high amount of messages for rebroadcast, the dissemination mechanism has to coordinate the message rebroadcast, depending on the context. A static re-transmission interval will not take into account the changing context. In addition, it does not allow for changing relevance, according to the message age and distance to the reported hazard.

Within the benefit function, a parameter incorporating the age of a message makes sure that vehicles transmit messages with higher relevance, if they are more up-to-date and more likely to be unknown to other vehicles due to their younger

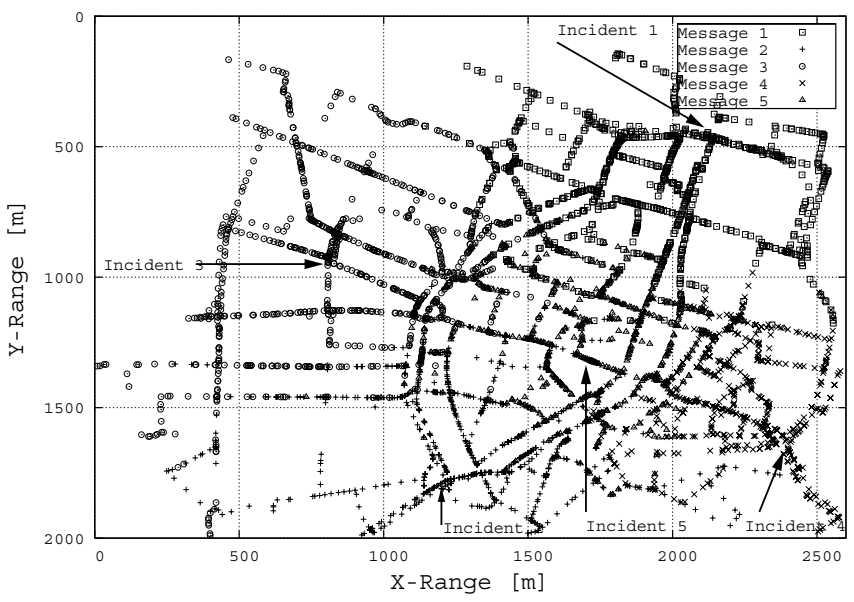

Fig. 6. Dissemination areas in a scenario with five hazards, each with an equal impact

age. As a result, the context-adaptivity of the message lifetime is increased.

Fig. 7 shows a scenario with an average vehicle density of 200 vehicles and three hazards that appear at different points in time during the simulation. The graph depicts the amount of additional message transmissions that were executed during the last $10 \mathrm{~s}$ of simulation time. It can be seen clearly that messages loose relevance as their age increases. As long as the vehicle has enough message storage capacity and available bandwidth, then an old message also has a chance to be transmitted. However, due to the artificially high bandwidth limitation in the simulation scenario, older messages were rapidly suppressed by new messages.

The self-organizing diffusion approach meets the requirement of context-adaptive message lifetime because the messages will be kept within the send queues. After a successful transmission, the relevance for an immediate re-transmission is usually very low. However, the relevance is likely to increase again, depending on the time that this particular message has not been broadcasted by surrounding nodes. This leads to an implicit retransmission as long as there is no additional message to be sent with a higher relevance value. Therefore, the likelihood of a successful and timely reception of the message by interested vehicles increases. At the same time, any retransmission will not delay any message that is currently time critical and more relevant. In addition, with increasing age, a retransmission of the old message gets more and more unlikely, until the message is dropped from the message queue due to limited storage capacity. Thus, the message lifetime is automatically regulated. By using certain thresholds for the message age, it can be implemented that messages are dropped from the message queue once reaching a certain age.

\section{Situation Adaptability}

Road situations are permanently changing; rain areas move, fog disappears, and accidents are cleared. The knowledge in vehicles should adapt to these changes in a manner as timely 


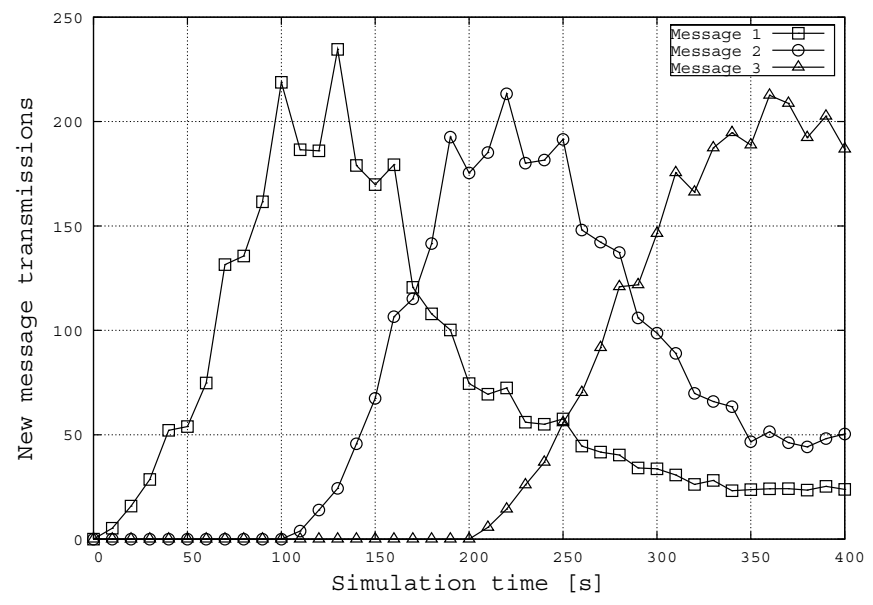

Fig. 7. Adapting message lifetime in a scenario with three hazards, each with a different time of appearance

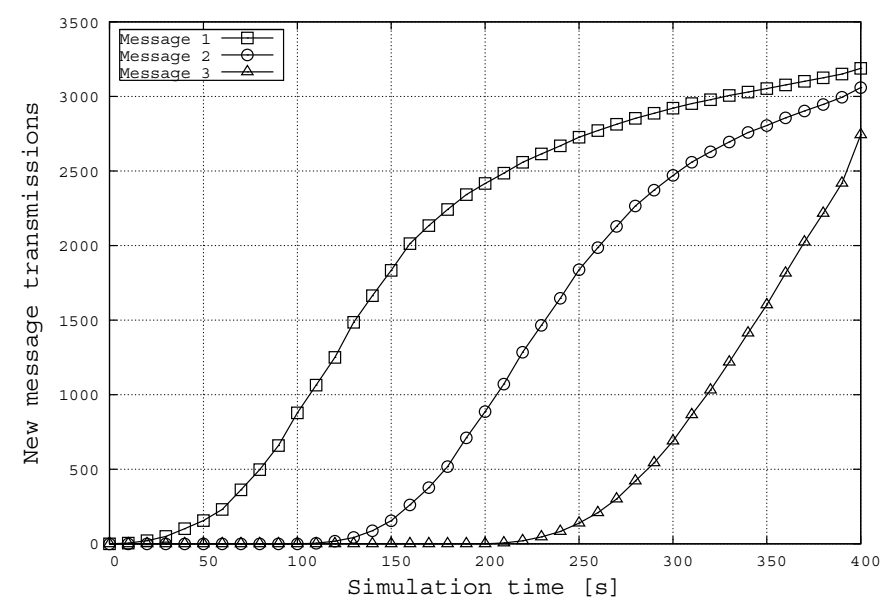

Fig. 8. Situation adaptability in a scenario with three hazards, each with a different time of appearance (Number of message transmissions)

as possible. In order to achieve that goal, it is necessary that the dissemination mechanism is able to adapt to the changing situation context quickly. This implies that the dissemination mechanism must be determined by the particular content of the message.

In the scenario in Fig. 8, three messages, each with different times of appearance, were chosen. It can be achieved that newer messages are disseminated faster and more frequent than older messages still existing at the same time. Therefore, they are capable of catching up in terms of dissemination among the vehicles.

\section{CONCLUSION}

In this paper we presented our concept for self-organized and context-adaptive information diffusion in VANETs. Due to the numerous possible hazard events many different warning messages will be broadcast simultaneously in future VANETs. Since data-rate and channel capacity is very limited in multihop networks, new strategies to reduce flooding intensity and message numbers have to be developed, to ensure the most relevant messages will be disseminated as fast as possible. In our concept we use utility evaluation for message content to select the messages most relevant to the overall network. By adapting the conventional medium access of 802.11, thereby influencing the channel access contention process, messages with higher relevance have a higher chance of being sent. Our simulation results showed that this method is capable of increasing the overall benefit.

In addition to changing medium access, defining dissemination areas and setting different message lifetimes can also lead to a more beneficial diffusion of warning information. Thereby, dissemination areas have to be determined in a self-organized fashion. Our simulation result showed, that self-organized message diffusion using context information is feasible and leads to dissemination areas automatically.

Using these two techniques - adapted medium access (influenced contention process) and self-organized message diffusion - helps to reduce the network load. In addition the most relevant messages for the participating nodes are sent first. This leads to the desired effect of optimizing the overall benefit for all network nodes. Hence, even in a loaded network the important messages can be distributed to the desired recipients with high speed.

\section{ACKNOWLEDGMENT}

This work has been carried out within the PReVENT WILLWARN integrated research project funded by the European Commission under the European VI Framework.

\section{REFERENCES}

[1] R. J. La and V. Anantharam, "Utility-based rate control in the internet for elastic traffic," in IEEE/ACM Transactions On Networking, 2002.

[2] M. Fazel and M. Chiang, "Nonconcave network utility maximization through sum of squares method," in Proceedings of the IEEE Control and Decision Conference, 2005.

[3] L. Wischhof, A. Ebner, H. Rohling, M. Lott, and R. Halfmann, "SOTIS - A Self-Organizing Traffic Information System," in Proceedings of the 57th IEEE Vehicular Technology Conference (VTC 03 Spring), Jeju, South Korea, April 2003.

[4] L. Wischhof and H. Rohling, "On Utility-Fair Broadcast in Vehicular Ad Hoc Networks," in Proceedings of the 2nd International Workshop on Intelligent Transportation (WIT 2005), Hamburg, Germany, March 2005.

[5] T. Kosch, "Situationsadaptive Kommunikation in Automobilen Ad-hoc Netzen,” Ph.D. dissertation, Munich University of Technology, 2005.

[6] C. Maihöfer, W. Franz, and R. Eberhardt, "Stored Geocast," in Proceedings 13. ITG/GI-Fachtagung Kommunikation in verteilten Systemen (KiVS), Leipzig, Germany, February 2003.

[7] C. Lochert, M. Mauve, H. Füßler, and H. Hartenstein, "Geographic Routing in City Scenarios," in ACM SIGMOBILE Mobile Computing and Communications Review (MC2R), January 2005.

[8] Y.-B. Ko and N. H. Vaidya, "Geocasting in mobile ad hoc networks: Loaction based multicast algorithms," in Proceedings of IEEE Workshop on Mobile Computing Systems and Applications, 1999.

[9] S. Eichler, C. Schroth, T. Kosch, and M. Strassberger, "Strategies for context-adaptive message dissemination in vehicular ad hoc networks," in Proceedings of the Second International Workshop on Vehicle-toVehicle Communications (V2VCOM 2006), July 2006.

[10] L. Briesemeister and G. Hommel, "Role-based multicast in highly mobile but sparsely connected ad hoc networks," 2000. [Online]. Available: citeseer.ifi.unizh.ch/briesemeister00rolebased.html 